Conclusion In a small cohort of children undergoing CPB surgery, increased coronary flow and, to less extent, prolonged CPB appeared to be associated with heightened risk for delayed myocardial repolarization after surgery.

\section{IMPACT OF CHYLOTHORAX ON THE POST OPERATIVE OUTCOME AFTER PEDIATRIC CARDIOVASCULAR SURGERY}

doi:10.1136/archdischild-2012-302724.1664

SR Ismail, M Kabbani, H Najm, G Shaath, A Jijeh. Cardiac Science, King Abdulaziz Medical City for National Guards, Pediatric Cardiac Intensive Care, Riyadh, Saudi Arabia

Background Chylothorax is the accumulation of Chyle in the pleural cavity, usually develops after disruption of the thoracic duct along its intra-thoracic route. In the majority of cases this rupture is secondary to trauma (including cardio thoracic surgeries). Chylothorax is a potentially serious complication after cardiovascular surgeries that require early diagnosis and adequate management.

Methods A retrospective study of all cases Complicated with Chylothorax after pediatric cardiac surgery in King Abdulaziz Cardiac Center between January 2007 and December 2009 was conducted. The study aim to determine the risk factors, and the impact on the post operative course.

Results We have 1135 cases operated during the study period, 57 cases (5\%) were complicated with chylothorax in the post operative period, The most common Surgeries complicated with chylothorax were the single ventricle repair surgeries (Glenn- Fon$\tan ) 15$ cases (27\%), followed by the arch repair cases 10 cases $(18 \%)$, the ventricular septal defect cases 10 cases (18\%), the Atrioventricular septal defect cases 7 cases (12\%), the arterial switch cases 6 cases $(11 \%)$, and others 8 cases (14\%). The ICU stay, the length of hospital stay and the bypass time were significantly longer in the chylothorax group, also the ventilation time, the inotropes duration and number were higher in the chylothorax group.

Conclusion Chylothorax after pediatric open heart surgery is not an uncommon complication, it Occurs more commonly with single ventricle repair and Aortic arch repair surgeries, it has a significant impact on the post operative course and post operative morbidity.

\section{NONINVASIVE MONITORING OF HEMOGLOBIN CONCENTRATION IN PEDIATRIC CARDIAC POSTOPERATIVE PATIENTS}

doi:10.1136/archdischild-2012-302724.1665

P García Soler, JM Camacho Alonso, A Morales Martínez, G Milano Manso. Pediatric Intensive Care Unit, Hospital Regional Universitario Materno Infantil Carlos Haya, Málaga, Spain

Background/Aim One of the major concerns after cardiovascular surgery is to detect bleeding and optimize hemoglobin $(\mathrm{Hb})$ to improve cardiac output, leading to repeated blood tests. We describe our experience with a new method of a noninvasive and continuous measurement of $\mathrm{Hb}$ concentration.

Methods Partial results of a prospective study in children after cardiac surgery, from January to March 2012. SpHb monitoring was performed during the first 48 hours after surgery (Pulse Co-Oximeter Radical-7TM/7.8.0.1, Masimo, CA). When each blood sample was taken, we collected the data from Radical- $7^{\mathrm{TM}}$, using central laboratory as a reference method (SiemensADVIA2120i). Data are reported as mean values and SD (normally distributed) and as median values and minimum-maximum range (distribution non-normal).

Results 78 blood samples were drawn from 21 patients, with a median age of 1.3 months (0.23-56), a median weigh of $3.7 \mathrm{~kg}$ (3-16.6) and a perfusion index(PI) of $1.2(0.21-13)$. All the measurements were performed in sedated subjects, $79.5 \%$ of them were intubated. Mean $\mathrm{Hb}$ on the laboratory analyzer was $12.47 \pm 2.28 \mathrm{~g} / \mathrm{dl}$ and mean $\mathrm{Hb}$ on the pulse oximeter $(\mathrm{SpHb})$ was $13.2 \pm 2.26 \mathrm{~g} / \mathrm{dl}$; its correlation coefficient was 0.75 and $\mathrm{R}^{2}$ was $0.55(\mathrm{p}<0.05)$. The mean of differences between both methods was $0.75 \pm 1.6 \mathrm{~g} / \mathrm{dl}$. BlandAltman plot shows that $65 \%$ had a difference $<1 \mathrm{~g} / \mathrm{dl}$ in comparison with laboratory hemoglobin.

Conclusions $\mathrm{SpHb}$ offers moderately acceptable accuracy in pediatric cardiac postoperative patients, maybe influenced by the low weight and PI of our patients. It has the advantages of providing continuous measurements, noninvasively, which may facilitate hemoglobin monitoring in the intensive care unit.

\section{INCIDENCE AND RISK FACTORS FOR POST CARDIAC SURGERY ARRYTHMIAS IN A TERTIARY PAEDIATRIC CARDIAC REFERRAL CENTRE}

doi:10.1136/archdischild-2012-302724.1666

${ }^{1} \mathrm{~K}$ Adhvaryu, ${ }^{2} \mathrm{R}$ Johnson, ${ }^{1} \mathrm{~N}$ Shetty. ${ }^{1}$ Paediatric Intensive Care Unit; ' ${ }^{2}$ Department of Cardiology \& Cardiothoracic Surgery, Alder Hey Children's Hospital, Liverpool, UK

Background and Aims Arrhythmias in the immediate postoperative period are a significant cause of morbidity. Its incidence varies between $15-79 \%$ and depends on case mix of surgical patients and methods used to identify them. Our aim was to determine the incidence and risk factors for their occurrence in our regional cardiac referral centre.

Methods A retrospective study was conducted including all paediatric (newborn to 18 years) patients who underwent a surgical procedure requiring cardio-pulmonary bypass between September 2010 and August 2011. Patients were identified and data gathered from the cardiac databases, electronic patient records and typed operation notes. Patients were excluded if they had pre-operative arrhythmias or if the arrhythmias during surgery were so transient such that they only occurred in the operation theatre.

Results Of the 346 cases that met inclusion criteria, 15\% developed arrhythmias of which half required intervention. Risk factors for arrhythmias included surgery involving the septum (21\% vs $8 \%$ non-septal), male (17\% vs $14 \%$ ) and age less than one year (20\% vs $9 \%)$. Those with arrhythmias had significantly longer bypass (160min vs $116 \mathrm{~min}, \mathrm{p}<0.05)$ and cross clamp times (101 min vs $77 \mathrm{~min}, \mathrm{p}<0.05)$. Transient rhythm problems that required intervention were sinus bradycardia, junctional rhythm, junctional ectopic tachycardia and supra ventricular tachycardia, whilst $43 \%$ of those with heart block required permanent pacing.

Conclusion Despite advances in surgical techniques arrhythmias after cardiac surgery were seen in a significant number $(15 \%)$ of our patients although only a small proportion $(<1 \%)$ require long term interventions.

\section{PERIOPERATIVE CHANGES IN RENAL BIOMARKERS IN CHILDREN UNDERGOING OPEN HEART SURGERY}

doi:10.1136/archdischild-2012-302724.1667

J Krastins, Z Straume, J Auzins. Pediatric Intensive Care, University Children's Hospital, Riga, Latvia

Introduction Cardiac surgery with cardiopulmonary bypass (CPB) is commonly perceived as a risk factor for decline in renal function. Hypothermia, hypoxia, hypotension, non-pulsatile blood flow during $\mathrm{CPB}$, use of $\mathrm{ACE}$ inhibitors, inotropic and (or) vasoactive support affects kidney and contributes to the acute kidney injury (AKI)

Objective To evaluate dynamics of renal biomarkers - serum creatinine (SCr) and glomerular filtration rate (GFR) in children undergoing open heart surgery.

Methods We conducted prospective, non-randomized observational study at the tertiary care University Children's Hospital Pediatric ICU. We enrolled 30 patients, 12 boys and 18 girls with CHD. Their median body weight was $6.8 \mathrm{~kg}$, (IOR $5.2<8.2 \mathrm{~kg})$ and median 
age 7 months (IOR $5<10$ months). SCr was determined and preoperative and postoperative creatinine clearance $(\mathrm{ClCr})$ was estimated using Schwarz formula. During surgical repair and till the end of the first 24 hours urine was collected to measure $\mathrm{ClCr}$, using the difference in urine (UCr) and SCr concentrations. Urine output, body temperature, duration of aortic cross clamping and cardiopulmonary bypass was recorded.

Results Median intraoperative urine output was $2.4 \mathrm{ml} / \mathrm{kg} / \mathrm{h}$ (IOR $1.29<3.15 \mathrm{ml} / \mathrm{kg} / \mathrm{h}$ ). Intraoperative $\mathrm{SCr}$ rised to $35 \mu \mathrm{mol} / \mathrm{l}$ (IOR $27.5<50.5$ ) vs preoperative SCr $29 \mu \mathrm{mol} / 1$ (IOR $24<32.9$ ), $\mathrm{p}<0,0001$. GFR declined from preoperative $98.4 \mathrm{ml} / \mathrm{min} . / 1.73 \mathrm{~m}^{2}$ (IOR $89.6<$ 123.04 ) to intraoperative $39.8 \mathrm{ml} / \mathrm{min} . / 1.73 \mathrm{~m}^{2}$, (IOR $24.9<65.5$ ), $\mathrm{p}<0,0001$

Conclusion Open heart surgery in children has severe, but transient effect on expression of renal biomarkers. Before discharge from the hospital both biomarkers returned to normal values.

\section{SYSTEMIC INFLAMMATORY RESPONSE SYNDROME (SIRS), MULTIPLE ORGAN DYSFUNCTION SYNDROME (MODS) AND SEPSIS AFTER OPEN HEART SURGERY IN CHILDREN}

doi:10.1136/archdischild-2012-302724.1668

${ }^{1} \mathrm{~T}$ da Rocha, ${ }^{2} \mathrm{~A}$ Botta, ${ }^{2} \mathrm{LD}$ Mulle, ${ }^{2} \mathrm{C}$ Ricachinevsky, ${ }^{1} \mathrm{P}$ Carvalho. ${ }^{1}$ Uti Pediatrica Hospital Santo Antonio, Universidade Federal do Rio Grande do Sul; '2Uti Pediatrica Hospital Santo Antonio, Santa Casa de Misericórdia de Porto Alegre, Porto Alegre, Brazil

Background and Aim Circulatory bypass (CBP) causes SIRS and MODS and the study of this prevalence is rare.

Methods We retrospectively made a cohort of 121 children operated during August, 2008 to July, 2009 to study the prevalence of SIRS, MODS and sepsis after open heart surgery.

Results The median age, weigtht, CBP time and temperature during CBP were 9 months, $6 \mathrm{Kg}, 55$ minutes and $32^{\circ} \mathrm{C}$, respectively. We found $48 \%$ of septal defects. During the first hour in PCIU 50.4 (\%) had the SIRS criteria with hypothermia present in $96.7(\%)$. Hypothermia was most frequent during all postoperative days except the second when hyperthermia was present in 41.6 (\%) vs $29.1(\%)$ of hypothermia. The others SIRS criteria such as elevated respiratory rate, heart rate and leucocytosis were present in 81.3 to $91.7(\%), 25$ to $37.5(\%)$ and 25 to $76.5(\%)$ of children, respectively. MODS criteria were present in 22.3 (\%) at arrive in PICU. During next postoperative days, MODS was more frequent than SIRS as follows: in the first day 53.8 (\%) vs $32.2(\%)$, second 41.3 (\%) vs 39.7 (\%), third 29.8 (\%) vs $19.8(\%)$, forth $24.8(\%)$ vs 17.4 (\%) and fifth $24.8(\%)$ vs $13.2(\%)$. After the third, forth and fifth postoperative days $56.2(\%), 62(\%)$ and $69.4(\%)$ of children, respectively, did not have SIRS or MODS. Prevalence of sepsis was 27.7 (\%).

Conclusion After open heart surgery in children, SIRS and MODS are more frequent in first 48 hours than other diagnosis.

\section{PERIPHERALLY INSERTED CENTRAL CATHETER (PICC) RELATED SUPERIOR VENA CAVA SYNDROME POST PATENT DUCTUS ARTERIOSUS LIGATION}

doi:10.1136/archdischild-2012-302724.1669

${ }^{1} \mathrm{M}$ Boyle, ${ }^{2} \mathrm{M}$ Cotter, ${ }^{1} \mathrm{~N}$ McCallion, 'D Corcoran, 'A Foran. ' Neonatology; ${ }^{2}$ Haematology, Rotunda Hospital, Dublin, Ireland

Background and Aims To assess incidence, management and outcomes of Superior Vena Cava (SVC) syndrome in post PDA ligation patients in the Rotunda Hospital. PICC's are used routinely in postoperative paediatric cardiac patients. Following placement, catheter-related thrombosis occurs in $8 \%$ to $45 \%$ of paediatric patients. Although uncommon, resultant SVC syndrome significantly complicates management of premature infants.

Methods A retrospective chart review of infants undergoing PDA ligation from July 2011 to March 2012.
Results 5 patients had PDA ligation within the study period. Average gestation at birth was $25+4$ weeks and average birth weight was $0.754 \mathrm{~kg}$. Surgery was performed at an average weight of $1.027 \mathrm{~kg}$ and 26.8 days. 9 PICC lines were inserted; mean of 1.8 per patient with removal following a mean of 12.5 days. 2 cases of catheter related thrombosis, post PDA ligation, resulted in SVC obstruction. Both patients had a PICC in situ at the time of surgery, the other 3 patients did not have PICC access during surgery. SVC thrombosis was detected at a mean of 15 days post operatively. One affected patient died subsequently due to complications.

Conclusions Post-surgical catheter related thrombosis is well documented. SVC syndrome can infrequently result as a complication, which may cause severe respiratory compromise leading to high morbidity and mortality. As treatment of SVC syndrome is very difficult, especially in post operative patients and with a trend towards fewer PDA ligations, increased awareness in neonatal units may allow early diagnosis and thrombolytic therapy to prevent the progression of this syndrome.

\section{INTERNET ADDICTION AMONG CHILDREN IN FAMILIES OF HIGH SOCIOECONOMIC LEVEL AND THE EFFECTS OF FAMILIAL FACTORS}

doi:10.1136/archdischild-2012-302724.1670

'SM Kayıran, ${ }^{2} \mathrm{TT}$ Comert, ' $\mathrm{N}$ Kose, 'B Gurakan. ' ${ }^{1}$ American Hospital; ' 2 Aydın University, Istanbul, Turkey

Background and Aims Internet use has increased rapidly in Turkey, raising concerns about physical and mental health problems associated with Internet addiction in adolescents. This study sought to describe Internet use among children aged 10-16 years in Turkish families of high socioeconomic level, and to evaluate the effects of familial factors.

Methods A questionnaire based on the Internet Addiction Test (IAT) was administered to 329 parents in American Hospital in Istanbul during the September-December 2010.

Results Of children and adolescents included in the sample, $45.3 \%$ (n: 149) were females, and 54.7\% (n: 180) were males. According the reports of parents, $9.1 \%$ ( $\mathrm{n}$ : 30 ) of children and adolescents were Internet addicts.

Conlusions We found that gender, age, and Internet use frequency affected Internet addiction. Although the prevalence of Internet addiction is low among Turkish children, preventive and educational initiatives and the involvement of addiction experts are necessary to reduce the effects of this emerging phenomenon.

\section{THE RELATIONSHIP BETWEEN SLEEP PATTERN AND OBESITY IN CHILDREN'S OF TEHRAN CITY}

doi:10.1136/archdischild-2012-302724.1671

${ }^{1} \mathrm{~A}$ Saber Gharamaleki, ${ }^{2} \mathrm{~A}$ Zamani, ${ }^{3} \mathrm{M}$ Hosseini, ${ }^{4} \mathrm{~S}$ Abedymanesh, ${ }^{5} \mathrm{~K}$ Jaafarian. 'Faculty of Health and Nutrition, Students' Research Committee, Tabriz University of Medical Science, Tabriz; '2Department of Nutrition and Biochemistry; ${ }^{3}$ Department of Epidemiology and Biostatistics, School of Public Health, Tehran; ${ }^{4}$ Department of Nutrition and Biochemistry, Ahvaz Jundishapur Medical Sciences University, Ahvaz; ${ }^{5}$ Department of Nutrition and Biochemistry, School of Public Health, Tehran University of Medical Science, Tehran, Iran

Objective To determine the relationship of sleep duration and efficiency with obesity in Tehran city children's.

Methods This study was conducted in Tehran city with 270 children aged 6 to 9 years. Heights, Weight, were measured and Body mass index (BMI), was calculated. Directly measured height and weight data were used to define overweight and obesity according to the centers for disease Control and Prevention's (CDC) 2000 Growth Charts. Nocturnal sleep duration and sleep efficiency were recorded by the Actigraphy method. 